\title{
Comunicación científica en el espacio digital. Acciones de difusión de proyectos de investigación del programa $\mathrm{H} 2020$
}

\section{Scientific communication in the digital space: actions for the dissemination of research projects under the $\mathrm{H} 2020$ program}

\author{
Manuel Gertrudix; Mario Rajas; Juan Romero-Luis; Alejandro Carbonell-Alcocer
}

How to cite this article:

Gertrudix, Manuel; Rajas, Mario; Romero-Luis, Juan; Carbonell-Alcocer, Alejandro (2021). “Comunicación científica en el espacio digital. Acciones de difusión de proyectos de investigación del programa H2020". Profesional de la información, v. 30, n. 1, e300104.

https://doi.org/10.3145/epi.2021.ene.04

Artículo recibido el 18-05-2020 Aceptación definitiva: 11-08-2020

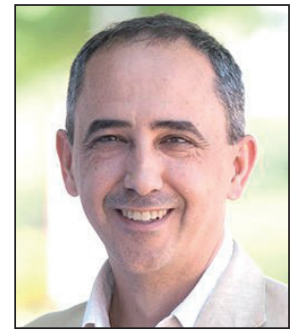

Manuel Gertrudix $\square$

https://orcid.org/0000-0002-5869-3116

Universidad Rey Juan Carlos

Facultad de Ciencias de la Comunicación Camino del Molino, 5 28934 Fuenlabrada (Madrid), España manuel.gertrudix@urjc.es

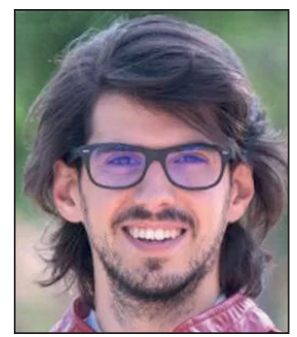

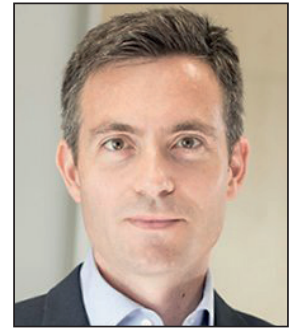

Mario Rajas

https://orcid.org/0000-0002-3807-8327

Universidad Rey Juan Carlos

Facultad de Ciencias de la Comunicación Camino del Molino, 5

28934 Fuenlabrada (Madrid), España

mario.rajas@urjc.es

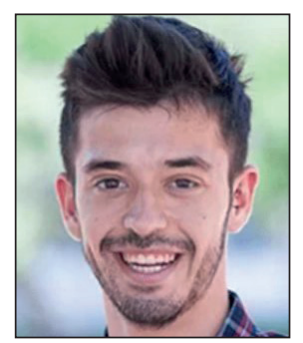

\section{Resumen}

La comunicación científica es un requerimiento exigido en las convocatorias competitivas de proyectos financiadas por la Unión Europea a través del programa Horizonte 2020. Los proyectos aprobados deben contar con un PEDR (Plan de explotación y diseminación de resultados) que establezca las estrategias de comunicación, y que concrete estas en acciones y productos que contribuyan a alcanzar a los diferentes grupos de interés atendiendo al modelo de la cuádruple hélice. En este artículo se analizan las acciones de divulgación y comunicación de los resultados de los proyectos de investigación del programa $\mathrm{H} 2020$, liderados por entidades, organismos o empresas de España, realizadas a través de la web y los medios sociales. La hipótesis de trabajo establece que las acciones de comunicación realizadas a través de la web y los medios sociales es escasa, estandarizada y responde a recursos y formatos de bajo coste y con producciones no profesionales. A partir de la base de datos Cordis de proyectos financiados por el programa $\mathrm{H} 2020$, se ha seleccionado una muestra de 50 proyectos elegidos en función del criterio de financiación. Se han elegido aquellos con un nivel más alto considerando que ello permitiría, en principio, una mayor dotación para las acciones de comunicación. La metodología aplica un análisis descriptivo y explicativo de las webs y los medios sociales utilizados por los proyectos mediante el Método de análisis de comunicación científica en medios digitales (Macomed), realizando una comparativa evolutiva entre 2017 y 2020. Las variables de análisis contemplan: a) la presencia en web y medios sociales, b) el nivel de utilización de estos canales de comunicación y difusión considerando métricas como el nivel de actividad, la diversidad de 
canales, la cadencia de publicación, el nivel de interacción con sus audiencias, el engagement de sus comunicaciones, y el nivel de producción que desarrollan. Los resultados muestran una comunicación en los medios digitales propios (web y redes sociales) que evoluciona con el desarrollo de los proyectos, pero que resulta desigual, con un alcance limitado fundamentalmente a Twitter y Facebook, y muy orientada a alcanzar a los pares de investigación y los agentes evaluadores, pero que carece de una orientación clara que cubra la demanda de abrir la comunicación a la sociedad tal como establecen los principios de la investigación e innovación responsable (RRI).

\section{Palabras clave}

Comunicación de la ciencia; Comunicación pública de la ciencia y la tecnología; RRI; Proyectos de investigación; Difusión de la ciencia; H2020; Horizonte 2020; Plan de comunicación; Redes sociales; Medios sociales; Medios propios; Medios digitales; Twitter; Facebook; Linkedln; YouTube.

\section{Abstract}

The European Union's H2020 program requires that all proposals submitted to calls include a scientific communication plan. Approved projects must have a plan for exploitation and dissemination of results (PEDR) that sets out the communication strategies. These must be translated into actions and products that contribute to reaching the different interest groups, following the four-helix model. This research analyzes the actions for disseminating and communicating the results of the research projects of the $\mathrm{H} 2020$ program led by entities, bodies, or companies in Spain and carried out through the web and social media. The hypothesis establishes that the communication actions carried out through the web and social media are scarce, standardized, and correspond to low-cost resources and formats, with nonprofessional production. From the database of projects funded by the $\mathrm{H} 2020$ program available on Cordis, a sample of 50 projects was selected based on funding criteria, choosing those with a higher level, as it is considered that this would, in principle, allow a greater allocation to communication actions. The methodology relies on descriptive and explanatory analyses of the websites and social media of the projects using the Method of analysis of scientific communication in digital media (Macomed), enabling an evolutionary comparison between 2017 and 2020. The variables of analysis are: (a) presence on the web and social media and (b) the level of use of these communication and dissemination channels considering metrics such as the level of activity, the diversity of channels, the rate of publication, the level of interaction with their audiences, the engagement with their communications, or the level of production achieved. The results reveal communication in digital media (web and social networks) that evolves with the development of the projects but that is unequal, with its scope limited mainly to Twitter and Facebook, and being highly oriented to reach research peers and evaluation agents, but lacking a clear orientation that addresses the demand for open communication to society as established by the principles of responsible research and innovation (RRI).

\section{Keywords}

Science communication; Public communication of science and technology; RRI; Research projects; Science dissemination; H2020; Horizon 2020; Communication plan; Social networks; Social media; Own media; Digital media; Twitter; Facebook; LinkedIn; YouTube.

\section{Financiación}

Investigación realizada con el apoyo del Ministerio de Ciencia, Innovación y Universidades de España dentro del proyecto "Comciencia: Comunicación eficaz, eficiente y responsable para proyectos de investigación competitivos" (CSO2017-82875-C2-1-R).

\section{Introducción}

El programa $\mathrm{H} 2020$ ha impulsado un cambio en el modelo de diseminación y divulgación científica de los proyectos financiados por la Unión Europea en programas competitivos al establecer, como un requerimiento exigido, que todas las propuestas incorporen un Plan de explotación y diseminación de resultados (PEDR) (Comisión Europea, 2016). Este plan debe establecer las estrategias de comunicación, concretándolas en acciones y productos que contribuyan a alcanzar a los diferentes grupos de interés. Ello para atender al modelo de la cuádruple hélice de la innovación -administración pública, sociedad civil, investigación y empresas- (Samelin, 2013), instaurar modelos de innovación más inclusivos, democráticos y eficientes (Braun; Starkbaum, 2019) contribuir a una gobernanza científica basada en investigación e innovación responsable (Comisión Europea, 2014a) y generar un impacto social medible (Ruvid, 2015).

Este nuevo enfoque solo puede llevarse a cabo mediante un proceso planificado de la comunicación científica, integrado desde el origen en el diseño de los proyectos, y que establece unos objetivos definidos, con la finalidad de alcanzar a unos públicos mediante un conjunto de estrategias de diseminación que hacen un uso óptimo de los canales y recursos de comunicación disponibles (Dudo; Besley, 2016; Gertrudix; Rajas; Gertrudis-Casado; Gálvez-de-la-Cuesta, 2020). Pero ello no presupone que, ante el mismo nivel de planificación, los resultados de comunicación, medidos en términos de impacto social, sean homogéneos. Como señala Flecha (2020), es fundamental diferenciar entre publicación científica, difusión, transferencia e impacto social, para orientar adecuadamente los esfuerzos de comunicación establecidos 
en el PEDR. La comunicación debe ser capaz de generar impacto social, mejorando las condiciones de vida de los ciudadanos y acercando los resultados a los decisores políticos para que sus políticas y toma de decisiones incorporen ese nuevo saber. La pregunta que surge es si las acciones que llevan a cabo los proyectos de investigación del programa H2020 están encaminadas adecuadamente a ello.
La comunicación debe ser capaz de generar impacto social, mejorando las condiciones de vida de los ciudadanos y acercando los resultados a los decisores políticos

El objetivo principal de la investigación es analizar las acciones de divulgación y comunicación de los proyectos de investigación del programa H2020, liderados por entidades, organismos o empresas de España, realizadas a través de medios propios. Para ello, se ha llevado a cabo un análisis de las webs de los proyectos, así como de la actividad en los principales medios sociales, realizando una comparativa evolutiva entre 2017 y 2020. La hipótesis principal de trabajo establece que las acciones de comunicación realizadas a través de la web y los medios sociales es escasa, estandarizada y responde a recursos y formatos de bajo coste y con producciones no profesionales.

\section{Estado del arte}

La necesidad de involucrar a todos los públicos en el conocimiento científico, de refuerzo y ampliación del diálogo entre ciencia y sociedad es una tendencia internacional que se ha ido consolidando a través de un lento proceso llevado a cabo en las últimas décadas que se ha unido, más recientemente, al concepto de ciencia abierta (Grand, 2020). El objetivo es establecer un compromiso público - public engagement- que permita implementar y estimular formas de comunicación que faciliten la interacción y la escucha, y ello genere un beneficio mutuo (National Coordinating Centre for Public Engagement, 2016). Un proceso de democratización que es tanto una necesidad como un derecho de la ciudadanía y que requiere, para Gallea (2017), comunicar la ciencia de manera muldireccional mediante un sistema de retroalimentación circular.

La obligatoriedad de incorporar planes de diseminación y divulgación en las propuestas de proyectos ha sido un elemento clave para impulsar este cambio de modelo. Como señala Berman (2017), los datos de impacto presentados en el marco de excelencia en la investigación de 2014 en el Reino Unido mostraron que la exigencia de incorporar en los proyectos un apartado de "vías de impacto" (pathways to impact) ha supuesto un notable incremento de las acciones de participación, lo que ha reforzado que esto se convierta en una acción estratégica tanto para los consejos de investigación como para las propias universidades. En esta línea, H2020 exige a los proyectos de investigación el diseño de planes de comunicación que contemplen de forma global la estrategia y las actividades de difusión, comunicación y explotación de resultados (Comisión Europea, 2014b). Dichos planes deben contemplar medidas estratégicas y especializadas para comunicar tanto el proyecto como sus resultados, identificar claramente la diversidad de audiencias a las que puede dirigirse, incorporando mecanismos bidireccionales cuando sea adecuado, y estableciendo alcances de los resultados de comunicación que sean medibles (European IPR Helpdesk, 2015; Scherer et al., 2018).

La evolución del entorno digital ha modificado la forma de abordar la comunicación de la ciencia, cobrando cada vez más importancia, en el modelo POE - paid, owned, earned- (Sciarrino et al., 2019) los medios propios -webs y redes sociales- y los medios ganados ${ }^{1}$ frente a los medios pagados, que suelen estar fuera del alcance natural de los presupuestos de los proyectos de investigación (López-Pérez; Olvera-Lobo, 2016).

La importancia de los canales sociales se ha visto impulsada por la facilidad de interacción entre los científicos, pero también de estos con los públicos a los que tratan de alcanzar con sus resultados (Collins; Shiffman; Rock, 2016) con la finalidad de aumentar los conocimientos científicos, promover la confianza, explorar opciones de carrera, establecer redes internacionales e influir en las políticas científicas (Yammine et al., 2018). A esta expansión ha contribuido el nuevo ecosistema móvil de comunicación (Valero-Pastor; García-Ortega, 2018) que facilita el acceso continuado a la información, pero también se enfrenta a problemas como la sobreinformación, la infoxicación y el desorden informativo que actúan como fuerzas de dispersión de la atención (Gertrudix-Barrio; Borges-Rey; García-García, 2017).

En los últimos años se han seguido dos líneas orientadas a optimizar la comunicación a través de los medios propios en el entorno digital, así como para tener una mayor capacidad de impactar en los medios ganados. Por una parte, la formación de los propios investigadores para mejorar sus habilidades como comunicadores científicos mediante manuales (Jamieson; Kahan; Scheufele, 2017), guías específicas (Cann, 2011) y cursos de formación (Baram-Tsabari; Lewenstein, 2017), y, por otra, mediante una creciente profesionalización de los procesos de comunicación a través de especialistas en la materia (Guenther, 2019).

Consecuentemente, los proyectos de investigación competitivos financiados en $\mathrm{H} 2020$ han convertido la utilización de medios propios tales como el sito web del proyecto o la comunicación en las principales redes sociales, como un 
activo fundamental de sus procesos de comunicación (Marín-González et al., 2017). El estudio de las webs de los proyectos ha sido abordada desde perspectivas diversas como el análisis del discurso (Lorés, 2020) o la incentivación de la participación a través de soluciones de colaboración (Fan, 2018), y la utilización de los medios sociales se ha interesado en el ecosistema de la denominada Ciencia 2.0 (Cabezas-Clavijo; Torre-Salinas; Delgado-López, 2009), el análisis de datos de los perfiles sociales para conocer las temáticas que se debaten, así como los enlaces que se comparten (Uhl; Kolleck; Schiebel, 2017) o el uso que hacen los centros especializados de investigación y las universidades públicas (López-Pérez; Olvera-Lobo, 2016; 2019).

\section{Metodología}

El modelo utilizado es el Análisis de Comunicación Científica en Medios Digitales (Macomed) (Gertrudix; Romero-Luis; Carbonell-Alcocer; Rajas, 2019) aplicado a los medios propios: web y canales sociales. La recogida de información se ha realizado mediante una ficha de análisis administrada a través de un formulario web.

Del procedimiento de análisis, que se describe de forma detallada en el apartado 3 del modelo Macomed, se han seleccionado para este estudio las preguntas de tres categorías, que se aplican como variables agrupadas:

- Presencia en webs y medios sociales,

- Nivel de utilización de los canales de comunicación, y

- Difusión e impacto en los principales canales de medios sociales.

Además, se incluyen las variables descriptivas de los proyectos para conocer su propósito, alcance, objetivo y públicos. En la descripción del modelo se incluye la relación completa de las variables descriptivas, las variables agrupadas, sus indicadores, sus valores y su respectiva operacionalización.

El análisis se ha llevado a cabo en un proceso longitudinal con dos tomas de registro: el primero en abril de 2017 y el segundo en marzo de 2020 con el fin de establecer una comparativa de la evolución de las variables. Las variables analizadas se han mantenido en ambos cortes temporales a excepción de las de la sección de noticias y suscripción o publicación de newsletter, que se incorporaron en 2020.

Para este estudio, el universo lo conforman los proyectos de investigación del programa H2020, liderados por entidades, organismos o empresas de España, que a 30 de abril de 2017, eran 1.340 según datos de Cordis. La muestra de este análisis está formada por los 50 proyectos con mayor financiación (Comisión Europea, 2017).

\section{Resultados}

\subsection{Presencia en web y medios sociales}

Los datos recogidos en relación a la variable agrupada Presencia en canales web y medios sociales reflejan un aumento significativo de 2017 a 2020 de los perfiles de YouTube/Vimeo (+20\%), de las webs (+10\%), de Twitter (+6\%), y de los canales LinkedIn $(+6 \%)$ como se puede observar en la figura 1. Otros canales también aumentaron, aunque menos: Facebook (+2\%) y Flickr (+2\%). En 2020 solo las presencias en Slideshare y Scribd se mantuvieron igual con respecto de 2017. Hay un grupo de medios sociales (Diigo, Delicious, Mendeley, Zotero, CiteULike, live streaming [Ustream...]) que no cuentan con presencia desde 2017.

En los proyectos analizados que cuentan con una web se ha identificado la presencia de blog, de sección de noticias y de apartados de suscripción o publicación de newsletters. En la recogida de datos de 2020, los autores identificaron la presencia de estas dos últimas y se procedió a su recopilación, pero no se cuenta con datos de la sección de noticias y suscripción o publicación de newsletter en 2017. Los datos de 2020 reflejan la presencia de la sección de noticias en 36 webs analizadas (75\%) y de newsletter en 14 (29\%) (figura 2). Estas últimas se representan de diferente forma: 10 casos en los que la newsletter está integrada en la web, 1 en el que solo se puede acceder mediante suscripción por email y 4 que cuentan con ambas opciones (integrada en la web y por medio de suscripción por email).

La presencia de blog es minoritaria. Solo se identifica en 5 de las webs, en 2017 y no se observan nuevos casos en 2020 , a pesar de que la presencia de webs aumentó un $10 \%$ de la muestra total.

\subsection{Nivel de utilización de los canales de comunicación y difusión}

\section{a. Variación del nivel de información de la web, blog y noticias, y frecuencias de actualización en blog, noticias y} newsletter

En términos generales, los datos recogidos en 2017 y 2020 reflejan muy poca variación entre el nivel de información tanto de la web como del blog. La diferencia que encontramos en el promedio del nivel de información de la web es poco sustancial ya que entre ambos periodos es de 2,74 (2017) y 3,1 (2020), correspondiente al valor "3. Bastante infor- 

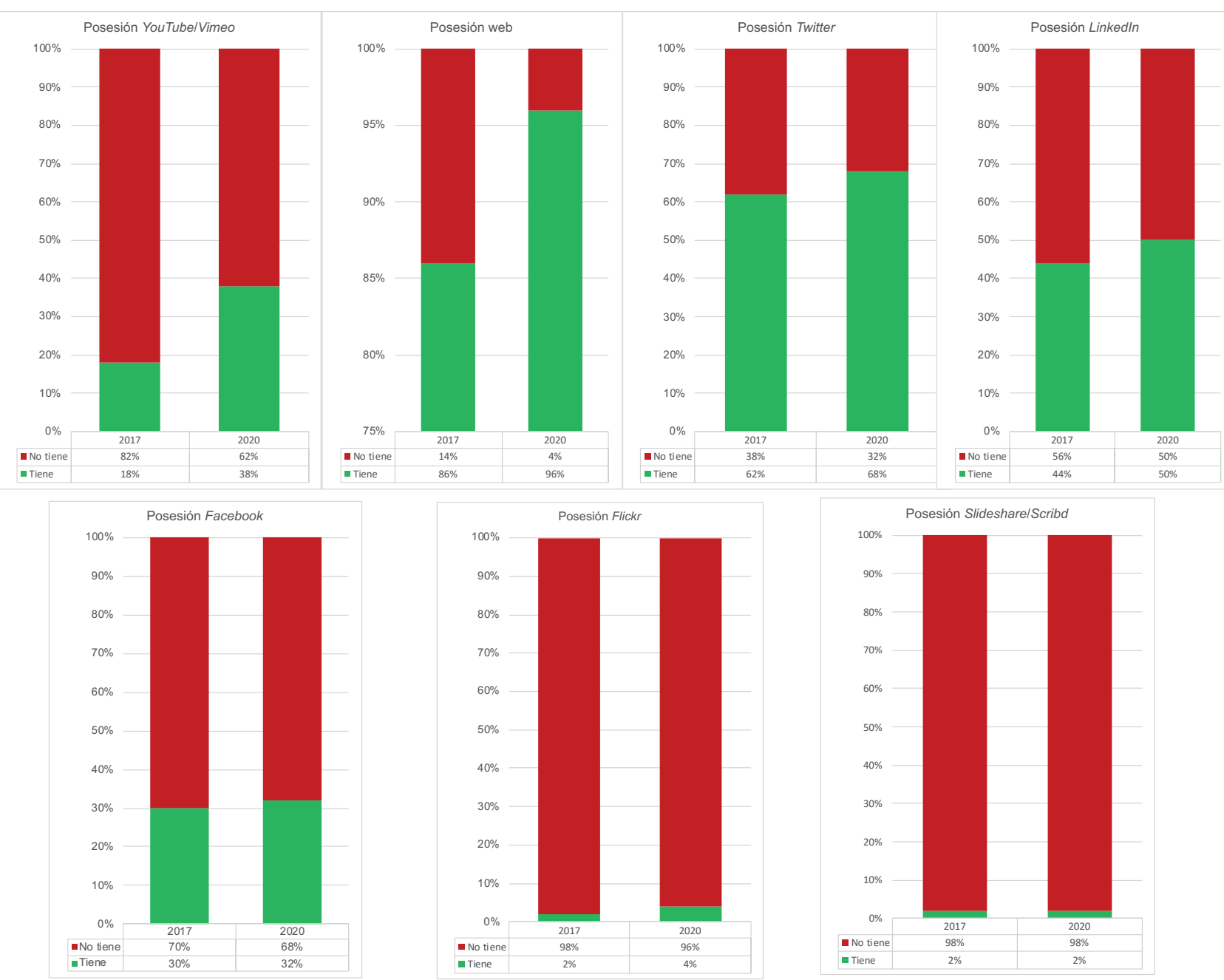

Figura 1. Evolución en porcentaje de la posesión de los medios sociales en 2017 y 2020 en toda la muestra.

mación" (figura 3). La desviación típica experimenta, también, una ligera variación, con valores que ascienden del 0,73 de 2017 al 0,75 de 2020.

A pesar de que no existe variación en el nivel de posesión de blogs entre 2017 y 2020, se produce un ligero cambio en el nivel de información disponible en estos entre ambos periodos. Tanto en 2017 como en 2020 un 80 \% de los blogs tienen poca información, si bien el 20\% restante pasa de encontrarse "no activo/en desarrollo" en 2017 a "tiene bastante información" en 2020.

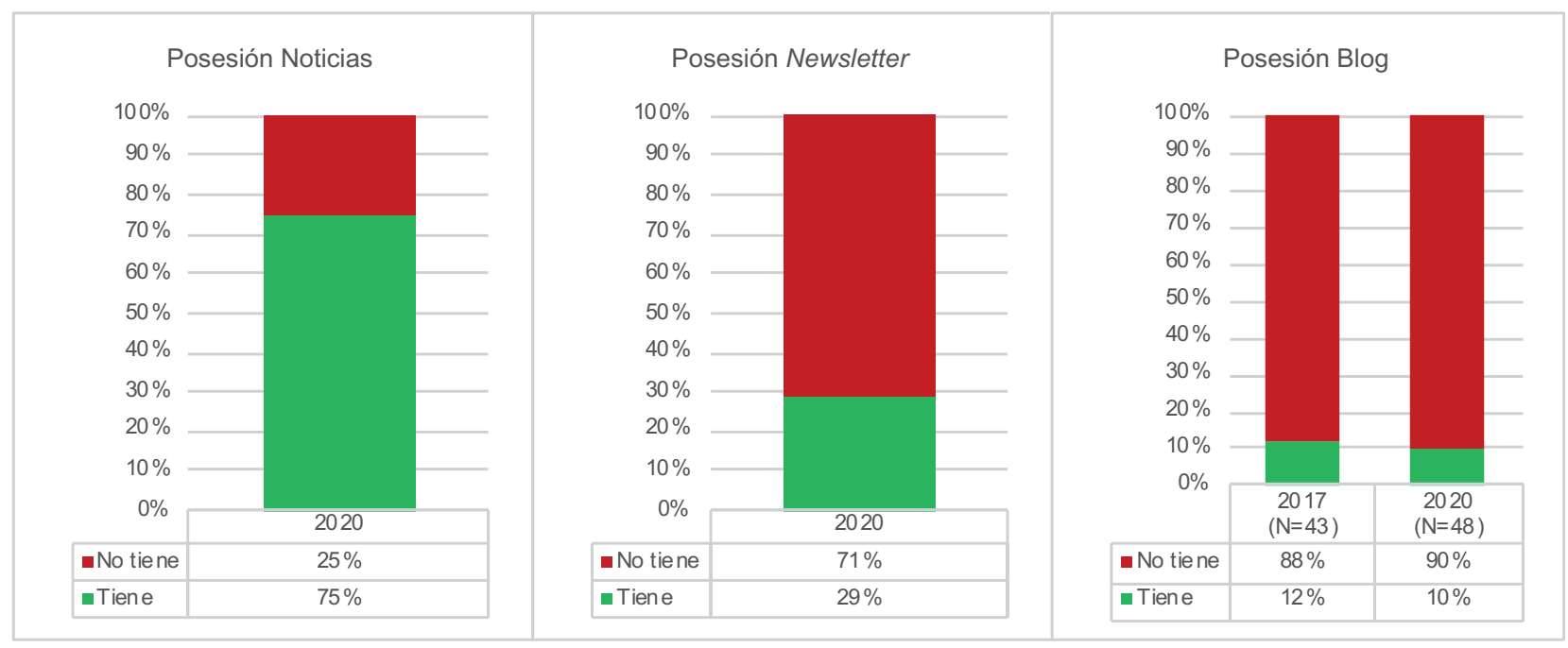

Figura 2. Porcentajes de posesión de blog, sección de noticias y newsletter con respecto al total proyectos que cuentan con web. 


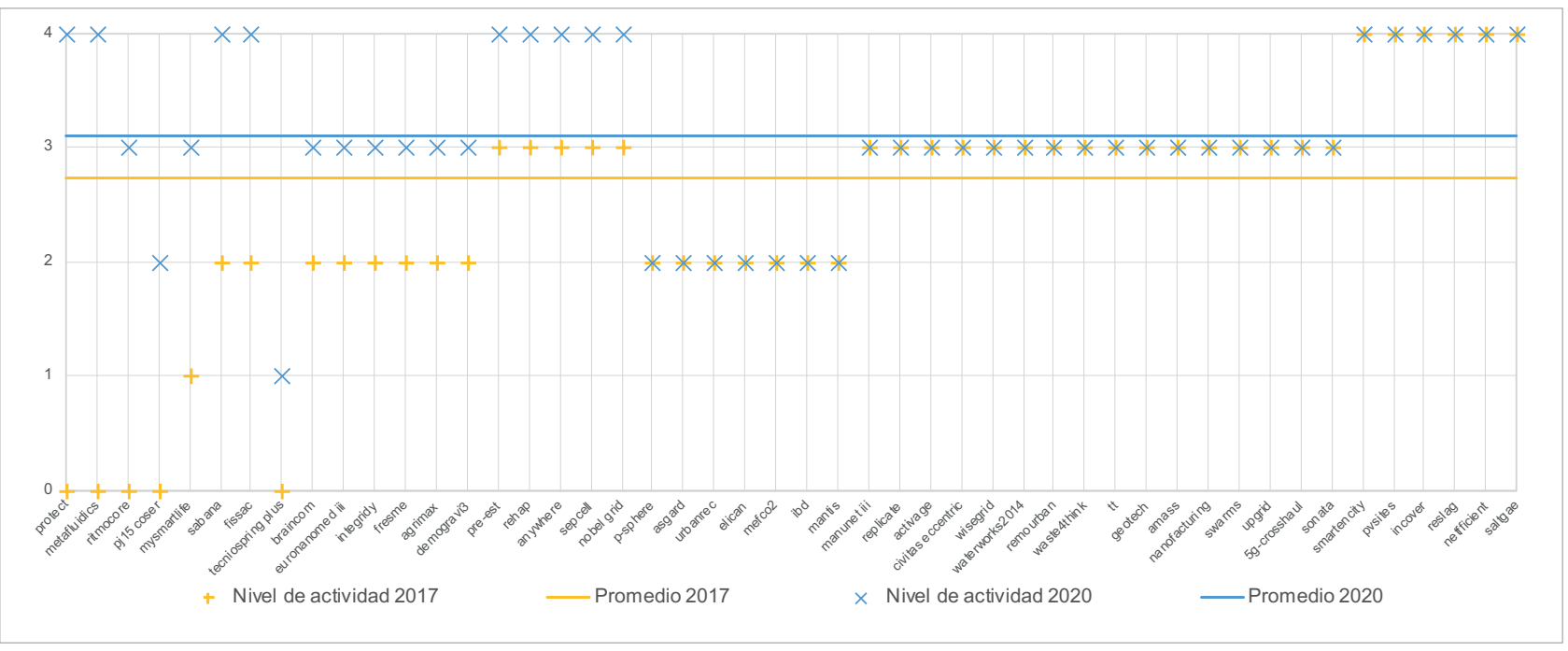

Figura 3. Variación del nivel de información de la web ordenada de mayor a menor variación.

Dado que no se cuentan con datos recogidos en 2017 del nivel de información de las noticias, se procede a una comparación entre los datos de esta variable entre la sección de noticias y blog (figura 4). Los datos revelan que el nivel de información en las noticias es mucho mayor que en el caso del blog. Hay que tener en cuenta, en cualquier caso, que el tamaño de la muestra $(\mathrm{N})$ en esta comparación está desequilibrado. Asimismo, se observa que, con respecto a la sección noticias, un $72 \%$ de las webs incluyen "bastante" o "mucha información", mientras que en el blog solo un $20 \%$ tienen "bastante información".

Con respecto a la frecuencia de actualización, si comparamos los datos recogidos de newsletter, noticias y blog en 2020 (figura 4), se observa que la frecuencia predominante es "esporádica" en las tres, con valores entre el $57 \%$ y el $40 \%$, lo que indica que la mayoría de las acciones de comunicación se realizan entre intervalos de más de un mes. Son pocos los casos donde la publicación es semanal o quincenal.

\section{b. Variación del nivel de información de canales de medios sociales}

En términos generales se observa un aumento del nivel de información de los principales canales de medios sociales (Twitter, LinkedIn, Facebook y YouTube) aunque de forma desigual (figura 5).

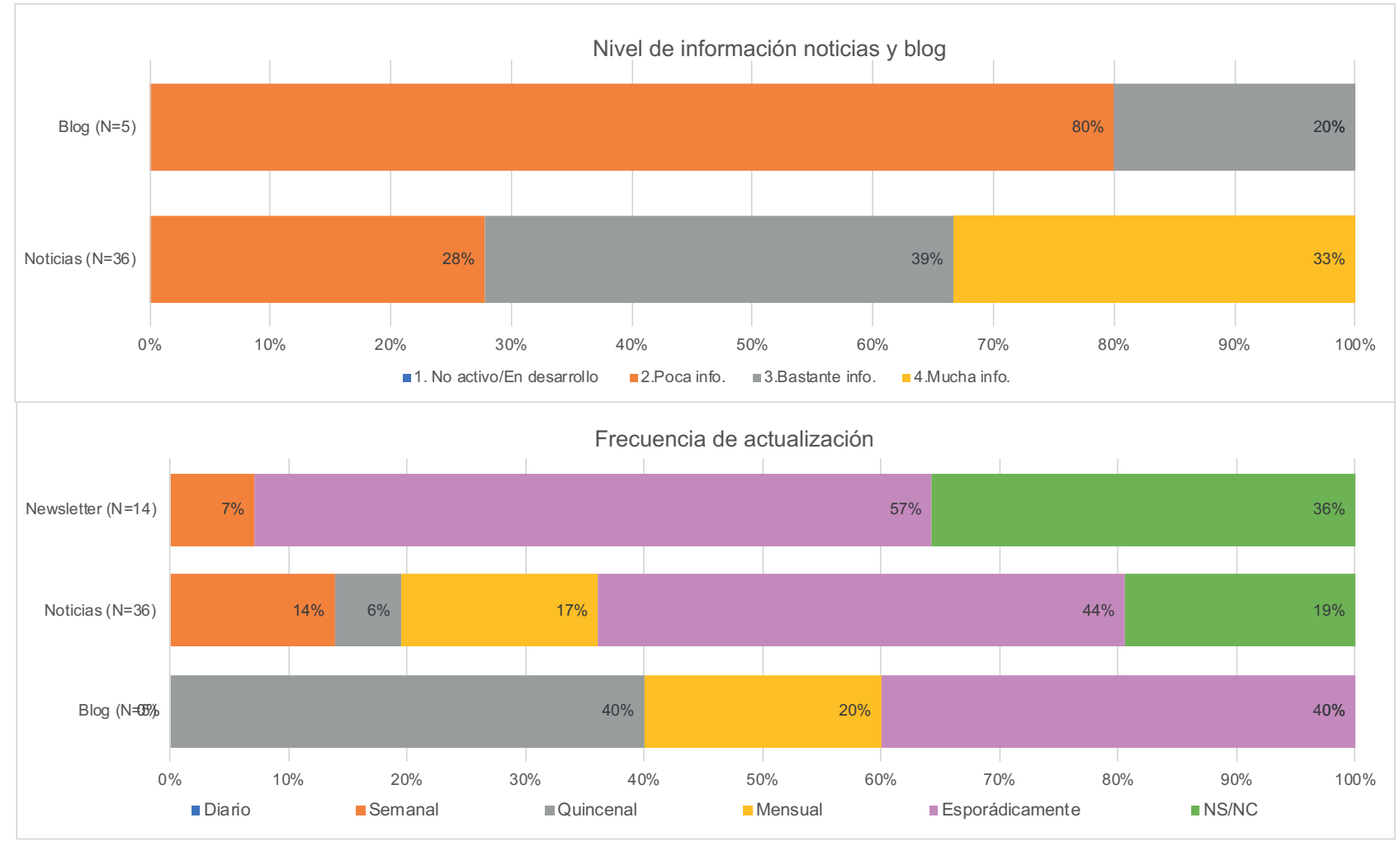

Figura 4. Nivel de información de noticias y blogs y frecuencia de actualización en 2020. 


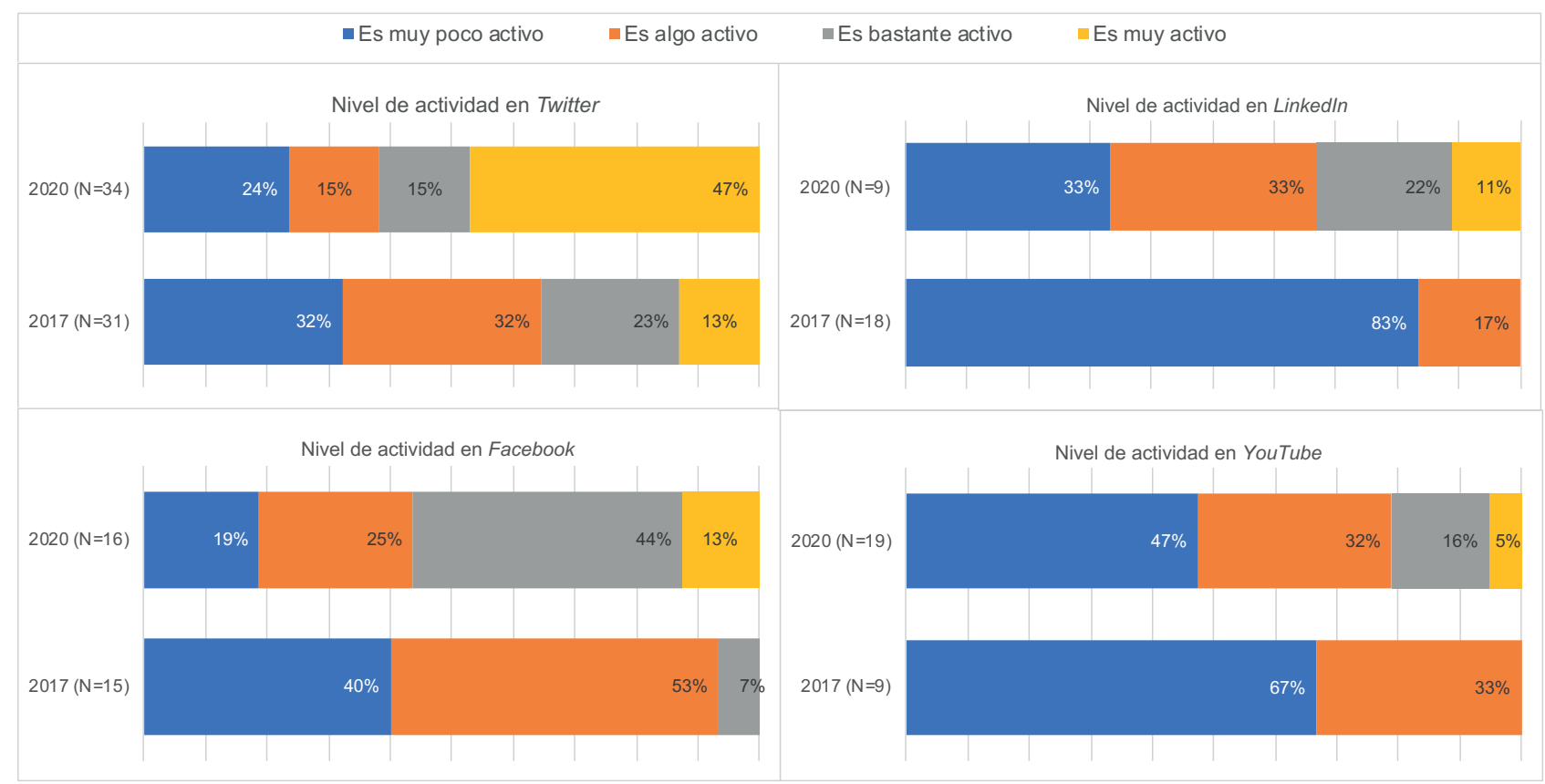

Figura 5. Variación del nivel de utilización de los principales canales de medios sociales.

Si bien en todos los casos el porcentaje del valor "es muy activo", este ha aumentado de 2017 a 2020, siendo en Twitter donde encontramos la mayor diferencia, con una subida del 34\%. Se trata de un aumento considerable teniendo en cuenta que el valor $\mathrm{N}$ aumenta de forma poco significativa (de 31 en 2017 a 34 proyectos con cuentas activas de Twitter en 2020).

Con respecto a Facebook se observa un aumento importante, aunque más moderado que el anterior, de un $13 \%$ para el valor "es muy activo". En 2017 no se identificó ninguna página con mucha actividad y, por tanto, este aumento es reseñable. También lo es el aumento del valor "es bastante activo" que crece un $37 \%$ entre ambos periodos.

En el caso de los datos que se han recogido con respecto a Linkedln merece la pena entrar con detalle. Lo más significativo es la reducción de la muestra entre $2017(\mathrm{~N}=18)$ y $2020(\mathrm{~N}=9)$. A pesar de que la muestra total de canales de $L$ inkedln es mayor, hay un gran número de cuentas de grupos privados que no se incluyen en el análisis de esta variable. Teniendo en cuenta este factor, hay una reducción del $50 \%$ para la valoración "es muy poco activo", lo que significa que en 2020 aumentan con respecto al 2017, ya sean las cuentas con "es algo activo" (+17\%), con "es bastante activo" (+22\%) y con "es muy activo" (11\%).

En el caso de YouTube el aumento es más moderado. Se debe tener en cuenta que el número de canales ha aumentado de 9 en 2017 a 19 en 2020. Consecuentemente, en 2020 aparecen los valores "es bastante activo" (+16\%) y "es muy activo" (+5\%), que en 2017 no alcanzaba ningún canal de YouTube.

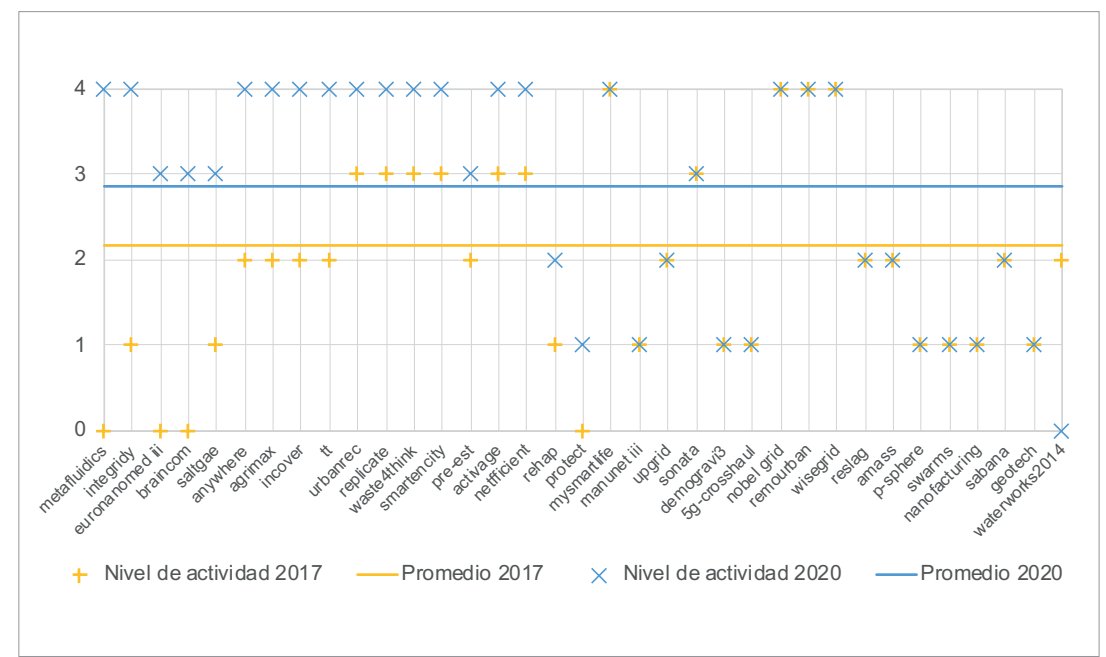

Figura 6. Variación del nivel de actividad de Twitter ordenada de mayor a menor variación.

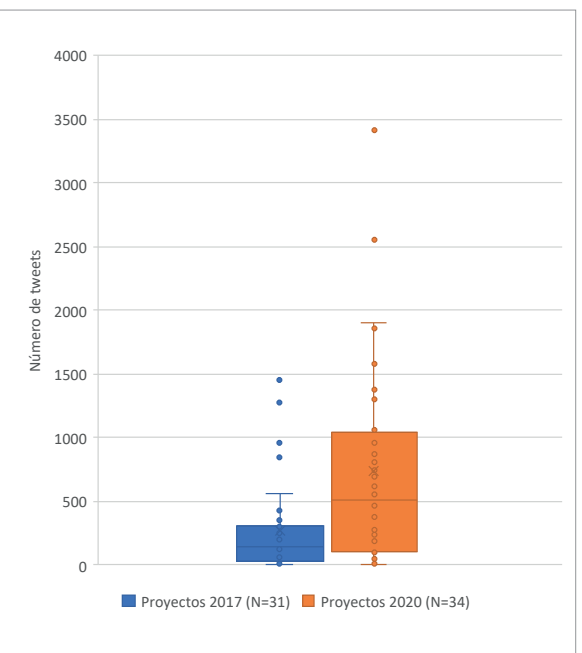

Figura 7. Número de tweets de las cuentas Twitter. 


\section{c. Variación del nivel de actividad de las cuentas de Twitter}

Se observa una variación sustancial con respecto a la actividad de Twitter. Su promedio aumenta de 2,16 en 2017 a 2,85 en 2020, pasando la media de "2. Algo activo" a "3. Bastante activas" (figura 6). El 45\% de los proyectos con cuentas de Twitter mantienen una utilización similar en el periodo, aunque se produce un aumento de proyectos que en 2020 disponen de una cuenta en esta red social. Conjuntamente, la desviación típica experimenta una variación de 0,22, pasando de 1,04 en el 2017 a 1,26 en el 2020.

El número de tweets totales emitidos refleja un valor interesante que ayuda a ilustrar de una forma más precisa el nivel de utilización de las cuentas de Twitter de los proyectos de investigación. Entre los dos años de recogida de información, se produce un incremento notable en la cantidad global de tweets, pasando la media de 273,06 en 2017 a 738,62 en 2020.

No obstante, es aún más interesante el análisis sobre el cambio de distribución del número de tweets entre ambos años. Los valores centrales de las dos distribuciones son 147 tweets en 2017 y 515,5 tweets en 2020. Encontramos una variación del rango intercuartílico de los valores de 2017, que asciende a 278,5, en 2017 mientras que en el año 2020 es de 940,25 . Se observa, por tanto, que en 2017 existe una gran concentración de proyectos cuyo número total de tweets se encuentra entre los valores 35 (Q1) y 313,5 (Q3) (15 proyectos), y que en 2020 hay concentración entre los valores 107,5 (Q1) y 1047,75 (Q3) (16 proyectos).

\section{d. Variación del nivel de utilización de las cuentas de Facebook}

El promedio en las cuentas de Facebook de los proyectos aumenta moderadamente pasando de 1,67 en 2017 a 2,5 en 2020. Es decir, este valor pasa de estar por debajo de "2. Es algo activo" a colocarse justo entre los valores "2. Es algo activo" y "3. Es bastante activo" (figura 8). Además, la desviación típica varía en 0,35, pasando de 0,62 en el 2017 a 0,97 en el 2020.

\section{e. Variación del nivel de utilización de las cuentas de LinkedIn}

Se observa un aumento de actividad de los canales LinkedIn en los perfiles y grupos públicos (18 en 2017 y 9 en el 2020). No obstante, merece la pena destacar el aumento elevado del porcentaje de canales privados, que pasa del $18 \%$ al $64 \%$.

Si bien podemos afirmar que el porcentaje de nivel actividad de las cuentas de los proyectos aumenta, no se cuenta con datos de la actividad de los grupos privados $y$, por tanto, no se puede afirmar que, teniendo en cuenta el total de la muestra de los canales LinkedIn, exista un aumento de la actividad entre la 2017 y 2020.

\section{f. Variación del nivel de utilización de las cuentas de YouTube}

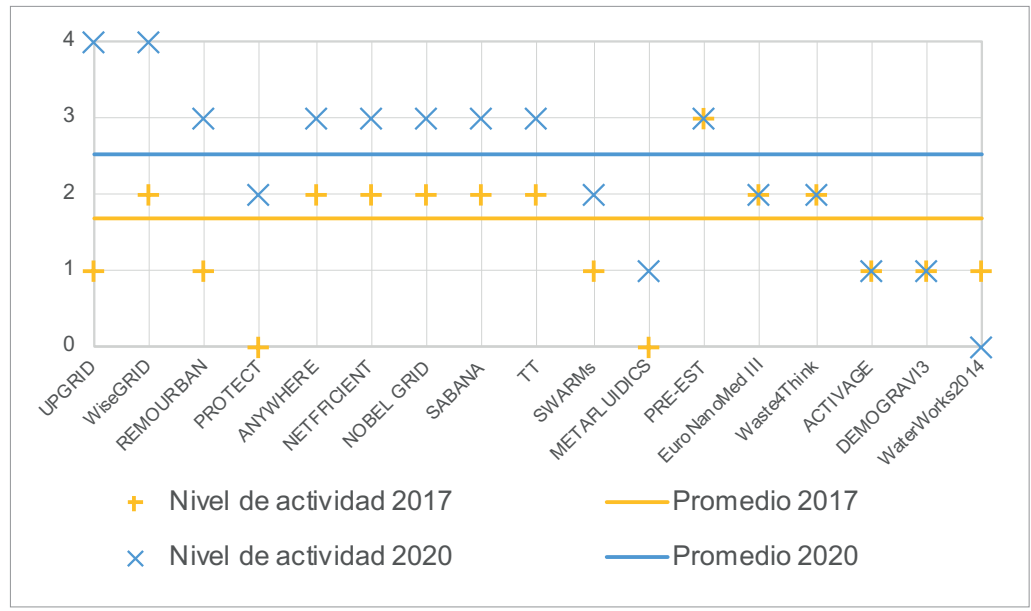

Figura 8. Variación del nivel de actividad de las cuentas de Facebook

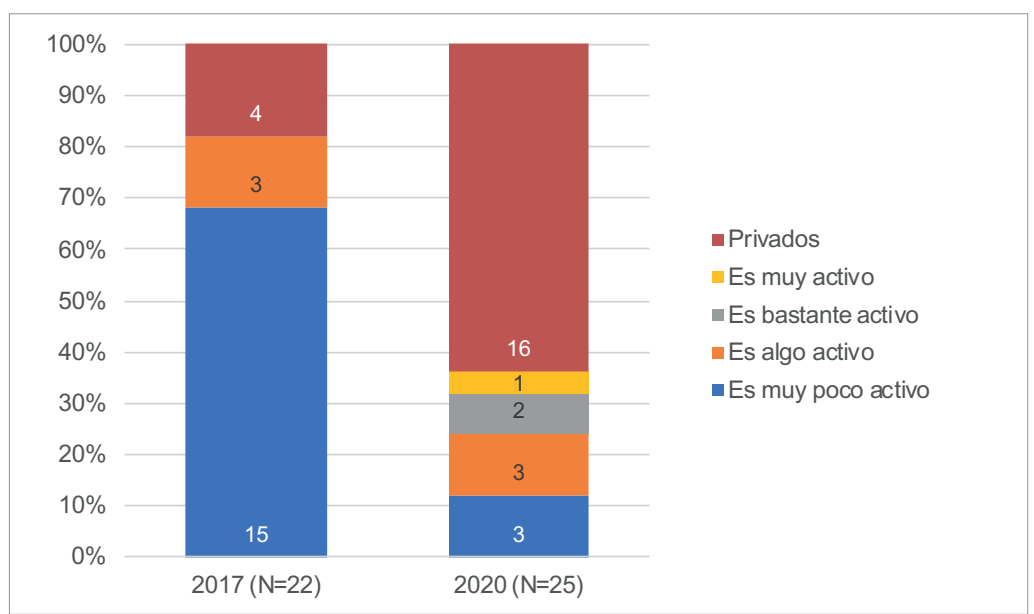

Figura 9. Variación en el nivel de actividad de las cuentas de Linkedln y canales privados.

La actividad en YouTube sigue la misma línea al alza que el resto de las canales de medios sociales. A pesar de que se trate del canal con menor actividad en ambos periodos (figura 5). El promedio de su nivel de actividad aumenta de 1,33 en 2017 a 1,79, en 2020. Redondeando las cifras, equivale a pasar de “1. Es muy poco activo" a "2. Es algo activo". Además, se produce una variación en la desviación típica de 0,42 en el nivel de actividad, pasando de 0,5 en 2017 a 0,92 en 2020.

El número de vídeos de los canales de las cuentas de YouTube que corresponden a los proyectos de investigación arrojan un valor que ayuda a comprender el nivel de utilización de dichos perfiles. Se observa un aumento evidente del número de vídeos publicados desde 2017 a 2020, que se concreta en una variación de la media de esta variable que asciende de 4,67 vídeos en 2017 a 23 vídeos en 2020.

Si se profundiza en el análisis, para observar la variación con respecto a la distribución, se comprueba que los valores centrales pasan de 2 en 2017 a 8 en 2020. Encontramos una variación considerable del rango intercuartílico, que ascien- 
de de 6 a 20 entre ambos años. Estas cifras marcan la evolución en uso de esta plataforma, que en 2017 era muy limitado, con la mayoría de los proyectos con un número total de vídeos de entre 2 (Q1) a 8 (Q3), y que en 2020 crece a valores entre los $8(\mathrm{Q} 1)$ y $20(\mathrm{Q} 3)$ vídeos totales.

\subsection{Impacto global obtenido en canales de medios sociales}

En términos generales, el impacto ha aumentado en 2020 con respecto al 2017. La diferencia más notable se observa en la variación media del número de seguidores de Facebook $(411,02)$ seguida de Twitter $(410,61)$, LinkedIn $(107,56)$ y YouTube $(25,43)$. Se trata de un dato relevante, ya que Facebook no se encuentra enfocada hacia el ámbito profesional. Además, para la valoración del impacto global, también se han analizado las variables que corresponden con el número total de likes de Facebook (figura 16) y el número de visualizaciones del vídeo con más reproducciones en los canales de YouTube (figura 17).

Con respecto al número de seguidores en Facebook, se observa un cambio notable en la distribución. Su recorrido intercuartílico pasa de 133,5 (2017) a 249,25 (2020). Destacan tres valores atípicos en 2020, entre los que se encuentra el proyecto que da el valor máximo de 3.431 seguidores (figura 12). Esas cifras son muy similares cuando se comparan con el número de likes (figura 16). La variación media del número de likes $(390,15)$ es menor que la variación media del número de seguidores $(411,02)$. En general, se encuentran pequeñas variaciones con respecto al número de seguidores y de likes en las mismas cuentas de $\mathrm{Fa}$ cebook.

Por otro lado, en la figura 13 se observa también una variación significativa con respecto a la distribución de los proyectos considerando el número de seguidores de las cuentas de Twitter, que varía considerablemente entre los dos periodos. La

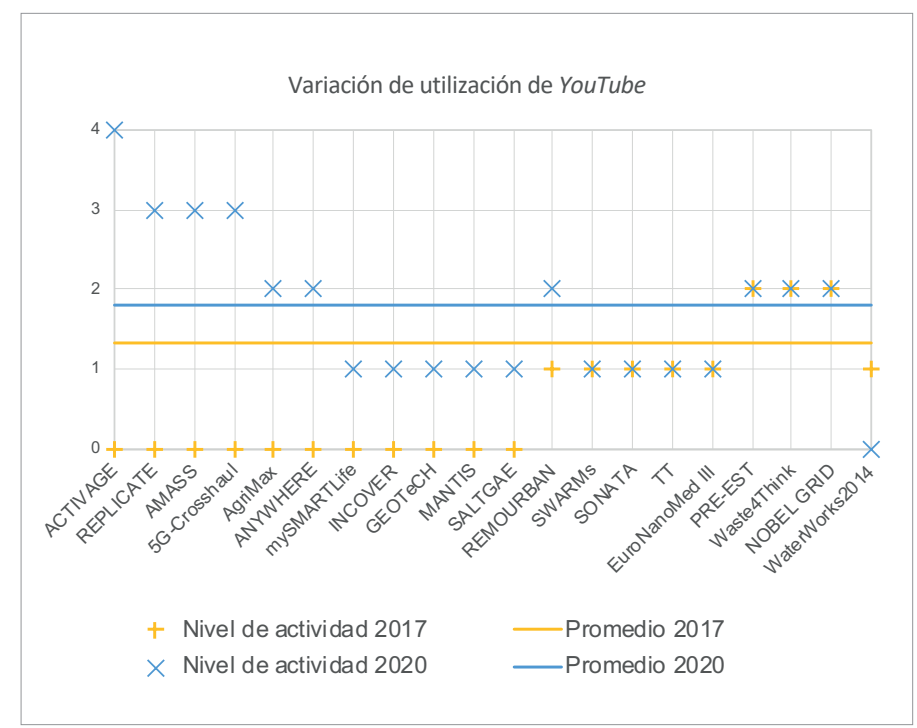

Figura 10. Variación del nivel de actividad de las cuentas de YouTube.

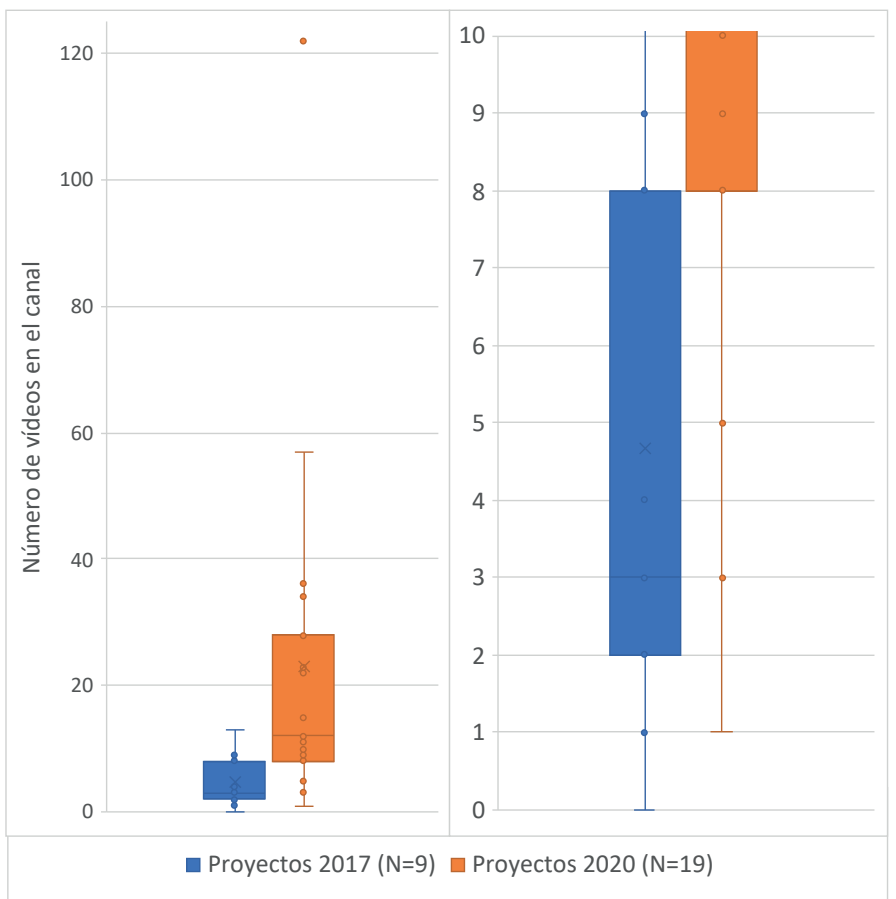

Figura 11. Número de vídeos en las cuentas YouTube.

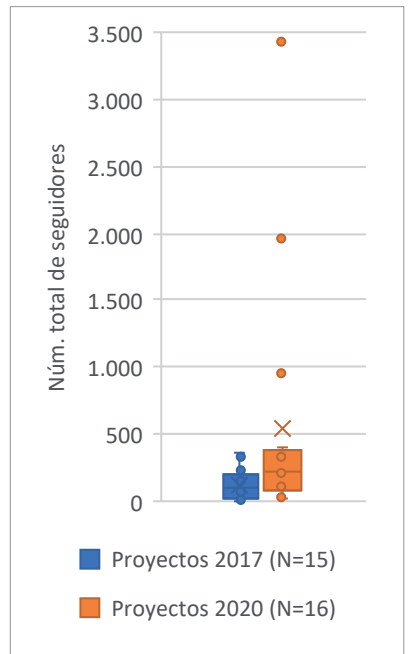

Figura 12. Impacto, seguidores Facebook.

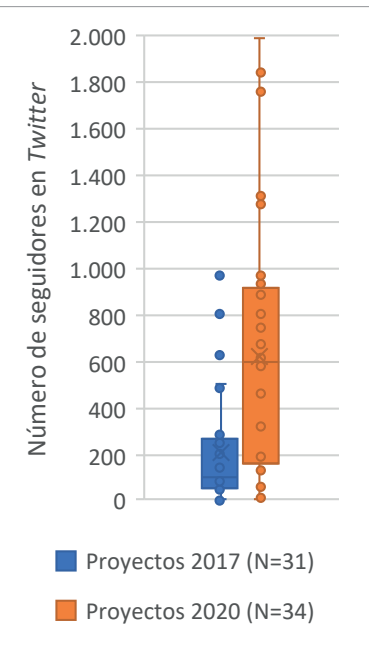

Figura 13. Impacto, seguidores Twitter.

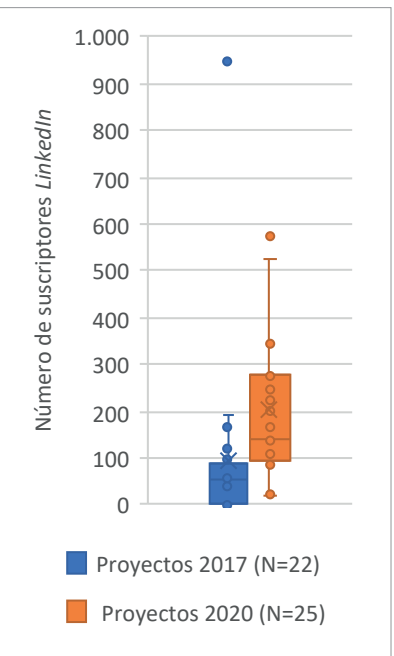

Figura 14. Impacto, seguidores Linkedln.

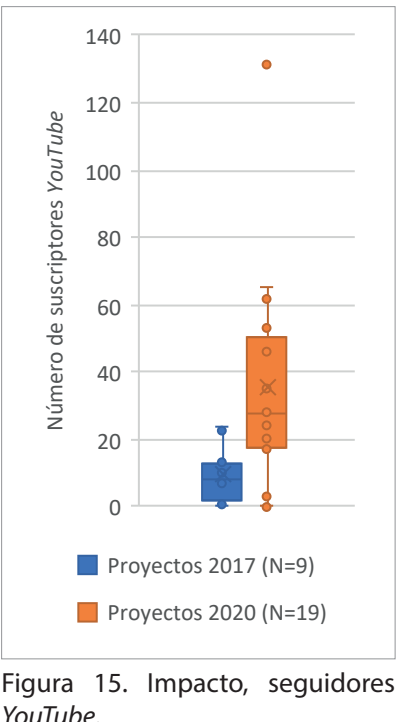

YouTube. 
mayoría de los proyectos se encuentran agrupados entre 51 (Q1) y 271 (Q3) seguidores en 2017, y entre 160 (Q1) y 922 (Q3) en 2020. Cabe destacar la existencia de valores atípicos tanto por encima del umbral Q3 (8 proyectos en 2017 y 9 en 2020) como por debajo del valor Q1 (8 proyectos en 2017 y 9 en 2020). Asimismo, la mediana varía notablemente, puesto que en 2017 (102) está muy cercana al valor Q1 y en 2020 (596) está más cercana al Q2.

Las redes sociales de Linkedln y YouTube son las que cuentan con menor impacto en relación con el número de seguidores. La red social para profesionales LinkedIn cuenta con un recorrido intercuartílico de 87,75 en 2017 y de 181 en 2020 (figura 14). El valor atípico que destaca en 2020, con 949, corresponde al caso WaterWorks2014. En 2017 la cuenta de LinkedIn estaba asociada exclusivamente al proyecto de investigación que forma parte de la muestra, pero en la recogida de 2020 se ha descartado ya que la cuenta ha integrado, además, a otros proyectos relacionados con la misma temática pero que no forman parte de este estudio.

En cuanto a YouTube destaca por ser la red social que cuenta con menos número de seguidores (figura 15). Sus valores cuentan con un recorrido intercuartílico de 11 en 2017 y de 33 en 2020. Un aspecto interesante, que arroja información sobre el impacto del canal de YouTube, es el número de reproducciones del vídeo con más reproducciones (figura 17). El vídeo con más reproducciones, en ambos periodos, es un valor atípico que se aleja del percentil 75 (1.381 en 2017 y 6.550 en 2020). El promedio de las reproducciones es muy variado, así como la distribución de este valor. La media en 2017 es de 373,22 y en 2020 de $1.472,68$, posiblemente debido a la diferencia de la $\mathrm{N}$ en los distintos periodos. Así, el rango intercuartílico es de 504 en 2017 y de 2.164 en 2020, lo que ilustra sobre el aumento considerable de las visualizaciones del vídeo con más reproducciones.

\subsection{Correlación entre financiación e impacto de los medios digitales propios}

El diagrama de dispersión muestra que no hay una relación directa entre la financiación global del proyecto y un mayor nivel de información en sus webs. El resultado del coeficiente de correlación es $r=-0,114$, lo que señala una correlación inversa y débil. Asimismo, se puede observar dos agrupaciones de proyectos con menor financiación que poseen un mayor nivel de información en los sitios web.

\section{Discusión y conclusiones}

El uso que se hace de los medios digitales propios es poco homogéneo y con un impacto limitado. El nivel de actualización de los blogs, newsletters y noticias es bajo, con ciclos de publicación mensuales y, en general, una baja actividad. Ello puede indicar que, a pesar del interés que estas aplicaciones despiertan, son posiblemente las que requieren un flujo de producción más constante y con mayor nivel de compromiso por parte de los miembros del proyecto para mantener una actividad continuada durante todo el ciclo de vida de este.

El nivel de información de los canales de medios sociales aumenta a medida que maduran los proyectos, aunque los resultados son heterogéneos entre estos. Es Twitter el medio que mayor actividad presenta y porcentualmente crece más, seguido de Facebook. LinkedIn tiene un seguimiento desigual y YouTube crece en volumen de actividad cuando el proyecto sigue una estrategia de comunicación basada en vídeo. Estos resultados son concordantes con los de otros estudios previos (Marín-González et al., 2017) que señalan cómo, a pesar del valor concedido a realizar una intensa comunicación a través de los medios propios (web del proyecto, uso de perfiles o hashtags en Twitter,
El uso que se hace de los medios digitales propios es poco homogéneo y con un impacto limitado. La actualización es baja, con ciclos de publicación mensuales 
Facebook, Linkedln...), tanto los centros de investigación y las universidades públicas (López-Pérez; Olvera-Lobo, 2016) como los proyectos financiados por el European Research Council (López-Pérez; Olvera-Lobo, 2019) hacen un escaso uso real de las redes sociales para la comunicación científica.
Se rechaza la hipótesis de que los proyectos con mayor presupuesto inicial concedido realizan acciones de diseminación en sus sitios web con mayor nivel de información

La mayor variación del nivel de actividad se produce, de manera consecuente, en Twitter, donde no solo aumenta el volumen sino que esta se distribuye de forma más homogénea entre todos los proyectos, lo que indica que para la mayor parte se convierte en un canal útil para la difusión de novedades y que, por tanto, se usa de forma habitual. Este aumento es más moderado en el caso de Facebook. En el caso de LinkedIn se produce un crecimiento importante de canales privados, lo que puede denotar el interés por mantener, en este canal, una comunicación de naturaleza especializada con pares o grupos de interés específicos relacionados con la temática del proyecto y con una divulgación de resultados menos abierta. En cuanto a YouTube, se produce un crecimiento en la intensidad de actividad vinculada al nivel de publicaciones de vídeos realizados, lo que es coherente con el nivel de maduración y desarrollo del proyecto, así como con la disponibilidad de recursos audiovisuales vinculados a este: promocionales, resultados, reuniones, acciones de difusión, etc. Ello indica que el uso de medios sociales para la comunicación de los proyectos se circunscribe a aquellos con mayor penetración social, como Twitter, Facebook, Linkedln y YouTube, con un uso definido para la distribución de novedades y la interacción, a veces muy contenida, con los seguidores de los perfiles o hashtags. Es residual el uso de otros servicios que, hace ya más de una década, se establecían como elementos del ecosistema de la ciencia 2.0 (Cabezas-Clavijo; Torres-Salinas; Delgado-López, 2009) tales como los blogs, los gestores de referencias y los sitios de etiquetado social.

En cuanto al impacto global obtenido en los canales, resulta interesante cómo Facebook, no siendo la red más activa, es la que logra un mayor aumento en el número de seguidores, lo que supondría que es la que resulta más eficiente en términos de esfuerzo/resultado. No obstante, Twitter sigue una evolución similar considerando tanto el número de seguidores como el de likes. LinkedIn y YouTube ofrecen las variaciones más modestas. En el caso de LinkedIn puede ser debido a que su carácter profesional hace que los aumentos sean más limitados, aunque tal vez de mayor calidad en términos de impacto a largo plazo. En el de YouTube, podría ser un indicador, sin embargo, de que la producción audiovisual realizada no despierta interés suficiente, pues los valores de reproducción son muy heterogéneos.

El análisis de correlación entre financiación e impacto de los medios digitales propios indica que no existe relación entre las variables correlacionadas, por lo que se rechaza la hipótesis de que los proyectos con mayor presupuesto inicial concedido realizan acciones de diseminación en sus sitios web con mayor nivel de información. Aunque no se pueden extrapolar las conclusiones al resto de la población, debido al tamaño de la muestra, sí se observa la tendencia de las variables para indicar que, por encima de un suelo de financiación mínimo para disponer de recursos necesarios para desarrollar profesionalmente las acciones de comunicación, un incremento de dicha financiación no comporta, necesariamente, un mayor impacto de estas.

Los resultados muestran una comunicación en los medios digitales propios (web y redes sociales) que evoluciona con el desarrollo de los proyectos, pero que resulta desigual, con un alcance limitado fundamentalmente a Twitter y Facebook, y muy orientada a alcanzar a los pares de investigación y los agentes evaluadores, pero que carece de una orientación clara que cubra la demanda de abrir la comunicación a la sociedad tal como establecen los principios de la investigación e innovación responsable (RRI).

\section{Nota}

1. Dentro del modelo de marketing POE (Paid, owned, earned), medios ganados son los espacios que se logran en medios de terceros de manera gratuita. Algunas acciones en comunicación científica estarían asociadas a las publicaciones, eventos, etc., que se obtienen en medios derivadas del envío de notas de prensa, acciones de RRPP, pero también mediante colaboraciones que se realizan con terceros publicando contenidos de forma periódica (reportajes, columnas, posts en blogs, contenidos audiovisuales en canales de YouTube, etc.).

\section{Referencias}

Baram-Tsabari, Ayelet; Lewenstein, Bruce (2017). "Science communication training: what are we trying to teach?”. International journal of science education, Part B, v. 7, n. 3, pp. 285-300. https://doi.org/10.1080/21548455.2017.1303756

Berman, David (2017). "Vote por engagement". THE Times Higher Education. https://www.timeshighereducation.com/opinion/vote-for-engagement 
Braun, Robert; Starkbaum, Johannes (2019). "Open forms of innovation collaborations should become more inclusive and democratic to make them efficient". RiConfigure.

https://irihs.ihs.ac.at/id/eprint/5356

Cabezas-Clavijo, Álvaro; Torres-Salinas, Daniel; Delgado-López-Cózar, Emilio (2009). “Ciencia 2.0: catálogo de herramientas e implicaciones para la actividad investigadora”. El profesional de la información, v. 18, n. 1, pp. 72-79. https://doi.org/10.3145/epi.2009.ene.10

Cann, Alan (2011). Social media. A guide for researchers. Department of Biology at the University of Leicester. http://www.rin.ac.uk/system/files/attachments/social_media_guide_for_screen_0.pdf

Carbonell-Alcocer, Alejandro; Romero-Luis, Juan; Gertrudix, Manuel; Rajas, Mario (2020). “Tablas de frecuencias. Acciones de difusión de proyectos de investigación del programa H2020 a través de medios propios" (versión 1) [data set]. Zenodo. http://doi.org/10.5281/zenodo.3785709

Collins, Kimberley; Shiffman, David; Rock, Jenny (2016). “How are scientists using social media in the workplace?”. PloS one, v. 11, n. 10, e0162680.

https://doi.org/10.1371/journal.pone.0162680

Comisión Europea (2014a). "Public engagement in responsible research and innovation". Portal H202O.

https://ec.europa.eu/programmes/horizon2020/en/h2020-section/public-engagement-responsible-research-and-innovation

Comisión Europea (2014b). "Dissemination \& exploitation of results". Fundig \& tender opportunities.

https://ec.europa.eu/research/participants/docs/h2020-funding-guide/grants/grant-management/dissemination-ofresults_en.htm

Comisión Europea (2016). "Communicating EU research and innovation guidance for project participants". Funding \& Tenders Portal.

https://ec.europa.eu/research/participants/docs/h2020-funding-guide/grants/grant-management/communication_en.htm

Comisión Europea (2017). Cordis. Resultados de investigaciones de la UE.

https://cordis.europa.eu

Dudo, Anthony; Besley, John C. (2016). "Scientists' prioritization of communication objectives for public engagement". PloS one, v. 11, n. 2, e0148867.

https://doi.org/10.1371/journal.pone.0148867

European IPR Helpdesk (2015). "Fact sheet. The plan for the exploitation and dissemination of results in Horizon 2020". IRP Helpdesk.

https://bit.ly/irphelpdesk-h2020

Fan, Chaonan (2018). "How to develop collaboration between bioeconomy clusters through a digital platform". Theseus.fi. https://www.theseus.fi/handle/10024/185287

Flecha, Ramón (2020). ¿Cómo conseguir o aumentar el impacto de los proyectos y cómo liderar un proyecto de referencia? How to improve the social impact of the projects?. Ars Civilis.

http://www.arscivilis.org/wp-content/uploads/2014/05/12_Ramon-Flecha_1de3.pdf

Gallea, José-Ignacio (2017). “¿Por qué debemos exigir al Estado la comunicación de la ciencia?”. La voz, 4 junio. https://www.lavoz.com.ar/opinion/por-que-debemos-exigir-al-estado-la-comunicacion-de-la-ciencia

Gertrudix-Barrio, Manuel; Borges-Rey, Eddy; García-García, Francisco (2017). “Redes sociales y jóvenes en la era algorítmica". Telos, v. 107, pp. 62-70.

https://telos.fundaciontelefonica.com/archivo/numero107/redes-sociales-y-jovenes-en-la-era-algoritmica

Gertrudix, Manuel; Rajas, Mario; Gertrudis-Casado, María-del-Carmen; Gálvez-de-la-Cuesta, María-del-Carmen (2020). "Gestión de la comunicación científica de los proyectos de investigación en H2020. Funciones, modelos y estrategias". Profesional de la información, v. 29, n. 4, e290424.

https://doi.org/10.3145/epi.2020.jul.24

Gertrudix, Manuel; Romero-Luis, Juan; Carbonell-Alcocer, Alejandro; Rajas, Mario (2019). “Modelo de análisis de comunicación científica en medios digitales - Macomed". Zenodo.

http://doi.org/10.5281/zenodo.3783778

Grand, Ann (2020). Open science and public engagement: Exploring the potential of the open paradigm to support public engagement with science. (thesis). University of the West of England.

https://uwe-repository.worktribe.com/output/941959

Guenther, Lars (2019). "Science journalism". Oxford research encyclopedia of communication.

https://doi.org/10.1093/acrefore/9780190228613.013.901 
Jamieson, Kathleen-Hall; Kahan, Dan M.; Scheufele, Dietram A. (eds.) (2017). The Oxford handbook of the science of science communication. Oxford University Press. ISBN: 9780190497620 https://doi.org/10.1093/oxfordhb/9780190497620.001.0001

López-Pérez, Lourdes; Olvera-Lobo, María-Dolores (2016). “Comunicación pública de la ciencia a través de la web 2.0. El caso de los centros de investigación y universidades públicas de España”. El profesional de la información, v. 25, n. 3. https://doi.org/10.3145/epi.2016.may.14

López-Pérez, Lourdes; Olvera-Lobo, María-Dolores (2019). “Participación digital del público en la ciencia de excelencia española: análisis de los proyectos financiados por el European Research Council”. El profesional de la información, v. 28, n. 1, e280106.

https://doi.org//10.3145/epi.2019.ene.06

Lorés, Rosa (2020). "Science on the web: The exploration of European research websites of energy-related projects as digital genres for the promotion of values". Discourse, context \& media, v. 35, 100389.

https://doi.org/10.1016/j.dcm.2020.100389

Marín-González, Esther; Malmusi, Davide; Camprubí, Lluís; Borrell, Carme (2017). "The role of dissemination as a fundamental part of a research project: Lessons learned from Sophie". International journal of health services, v. 47, n. 2 , pp. 258-276.

https://doi.org/10.1177/0020731416676227

National Coordinating Centre for Public Engagement (2016). "What is public engagement?". University of Bristol. https://www.publicengagement.ac.uk/about-engagement/what-public-engagement

Ruvid (2015). Impacto en los proyectos H2020. Recomendaciones para elaborar una propuesta. http://ruvid.org/ri-world/wp-content/uploads/2015/07/Guia_ImpactoH2020.pdf

Salmelin, Bror (2013). "The Horizon 2020 framework and open innovation ecosystems". Journal of innovation management, v. 1, n. 2, pp. 4-9.

https://doi.org/10.24840/2183-0606_001.002_0002

Scherer, Jörg; Weber, Sthephanie; Azofra, Maria; Ruete, Alexandra; Sweeney, Eugene; Weiler, Nina; Sagias, loannis; Haardt, Jakob; Cravettp, Rossella; Spichtinger, Daniel; Ala-Mutka, Kirsti (2018). "Making the most of your H2020 project". The European IPR Helpdesk project.

https://www.iprhelpdesk.eu/sites/default/files/EU-IPR-Brochure-Boosting-Impact-C-D-E.pdf

Sciarrino, JoAnn; Friedman, Jim; Kirk, Todd; Kitchings, Kim S.; Prudente, John (2019). “Customer journey modelling: An integrated approach to quantify the importance, contribution and efficiency of paid, owned and earned media". Parvatiyar, Atul; Sisodia, Rajendra (eds.). Handbook of advances in marketing in an era of disruptions: Essays in honour of Jagdish N. Sheth. SAGE. ISBN: 9789352809585

Uhl, André; Kolleck, Nina; Schiebel, Edgard (2017). "Twitter data analysis as contribution to strategic foresight - The case of the EU research project "Foresight and modelling for european health policy and regulations" (Fresher)". European journal of futures research, v. 5, n. 1, art. 1.

https://doi.org/10.1007/s40309-016-0102-4

Valero-Pastor, José-María; García-Ortega, Alba (2018). "La importancia de comunicar la ciencia en el nuevo ecosistema móvil”. Divulgación científica e innovación mediática. Comunicar la ciencia en el ecosistema móvil, p. 9. Egregius Ediciones. ISBN: 9788417270704

Yammine, Samantha Z.; Liu, Christine; Jarreau, Paige B.; Coe, Imogen R. (2018). "Social media for social change in science". Science, v. 360, n. 6385, pp. 162-163.

https://doi.org/10.1126/science.aat7303

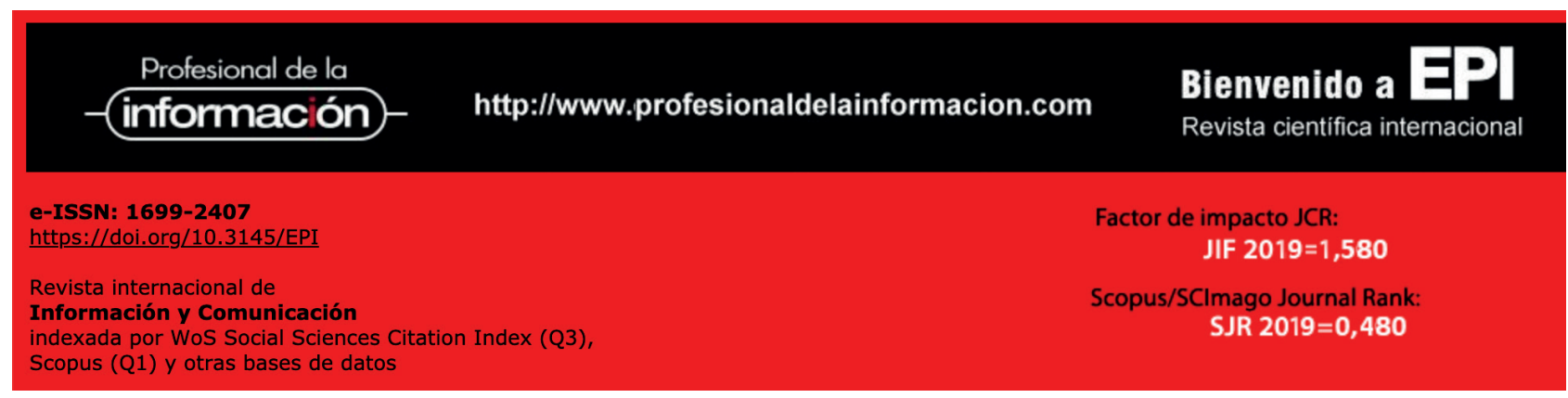

